\title{
Ground and airborne methane measurements with an optical parametric amplifier
}

\author{
Kenji Numata $^{1, a, b}$, Haris Riris ${ }^{b}$, Steve $\mathrm{i}^{\mathrm{b}}$, Stewart $\mathrm{Wu}^{\mathrm{b}}$, Stephan R. Kawa ${ }^{\mathrm{b}}$, Martha Dawsey ${ }^{\mathrm{b}}$, \\ Anand Ramanathan ${ }^{\mathrm{c}, \mathrm{b}}$, Jianping Mao ${ }^{\mathrm{b}}$, Michael Krainak ${ }^{\mathrm{b}}$, James Abshire ${ }^{\mathrm{b}}$ \\ a Dept. of Astronomy, Univ. of Maryland, College Park MD, USA 20742 \\ ${ }^{b}$ NASA Goddard Space Flight Center, Greenbelt MD, USA 20771 \\ 'Oak Ridge Associated Universities, Oak Ridge, TN, USA 37830
}

\begin{abstract}
We report on ground and airborne atmospheric methane measurements with a differential absorption lidar using an optical parametric amplifier (OPA). Methane is a strong greenhouse gas on Earth and its accurate global mapping is urgently needed to understand climate change. We are developing a nanosecond-pulsed OPA for remote measurements of methane from an Earth-orbiting satellite. We have successfully demonstrated the detection of methane on the ground and from an airplane at $\sim 11-\mathrm{km}$ altitude.
\end{abstract}

Keywords: Optical Parametric Amplifier, Lidar, DIAL, Space Instrumentation

\section{INTRODUCTION}

Methane $\left(\mathrm{CH}_{4}\right)$ is the second most important greenhouse gas on Earth and its atmospheric concentration has almost tripled since pre-industrial times [1]. Though its atmospheric abundance is less than carbon dioxide (1.78 ppm vs. 380 $\mathrm{ppm}$ ), it has a much larger greenhouse heating potential [2]. Methane also contributes to pollution in the lower atmosphere through chemical reactions leading to ozone production. Natural sources of methane include wetlands, wildfires, and termites, and perhaps other unknown but significant sources [3]. Recent observations have indicated that methane levels are on the rise as a result of increased fossil fuel production, rice farming, livestock, and landfills. Accurate knowledge of methane distribution and emissions is imperative for a correct assessment of its impact on global change [4]. Currently, our observations of greenhouse gases are limited to in-situ (surface and tower sites) and sparse airborne measurements. Accurate global methane observations are urgently needed in order to better understand climate change and reduce uncertainty in the carbon budget. Initial space measurements of methane are starting to become available from the JAXA (Japan Aerospace Exploration Agency) GOSAT (Greenhouse gases Observing SATellite) mission [5]. The MERLIN (Methane Remote Lidar Mission) on a small satellite has been proposed by DLR (German Aerospace Center) and CNES (National Center for Space Studies) in the frame of a German-French climate monitoring initiative [6].

At the NASA Goddard Space Flight Center (GSFC), we are developing a laser transmitter system for atmospheric methane measurements from an Earth-orbiting satellite. Our system is based on a nanosecond-pulsed optical parametric amplifier (OPA). An OPA is suited for this application since it can amplify a seed laser within the widely tunable gain bandwidth of the nonlinear crystal. Methane has an overtone absorption band at $1.6 \mu \mathrm{m}$, which has suitable strength for lidar measurement from space. The two laser sources an OPA needs, a 1064-nm Nd:YAG pump laser and a $1.6 \mu \mathrm{m}$ semiconductor seed laser diode (LD), are both mature technologies and can readily be applied to spaceflight. Through the ground and airborne demonstrations shown in this paper, an OPA proved to be a very promising approach for the spaceborne measurement of methane, and possibly, numerous other gases. To our knowledge, the demonstrated 11-km altitude is the highest to date for a methane airborne lidar, while measurements from helicopters ( $200-\mathrm{m}$ altitude) have been performed by various groups (see, for example, [7]).

\footnotetext{
${ }^{1}$ kenji.numata@nasa.gov; phone 1301-286-0799
}

Laser Radar Technology and Applications XVII, edited by Monte D. Turner, Gary W. Kamerman, Proc. of SPIE Vol. 8379, 83790H · C 2012 SPIE · CCC code: 0277-786X/12/\$18 · doi: 10.1117/12.918100 


\section{EXPERIMENTAL SETUP}

\subsection{OPA setup overview}

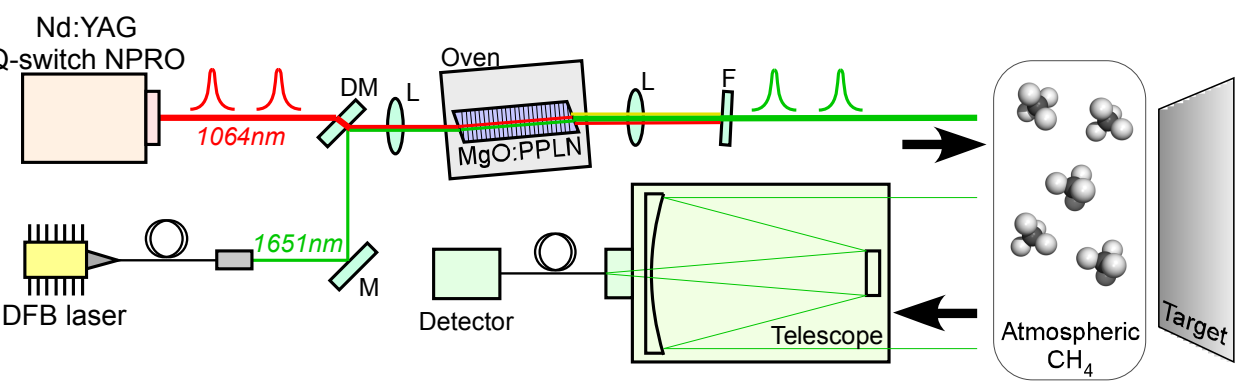

Figure 1. Conceptual diagram of the setup of a methane lidar based on an OPA. DM: dichroic mirror, M: mirror, L: lens, F: filter.

Figure 1 conceptually shows the experimental setup of the OPA lidar system for the measurement of atmospheric $\mathrm{CH}_{4}$. A nonlinear crystal was pumped by a pulsed $1064 \mathrm{~nm}$ laser and seeded by a continuous-wave distributed-feedback (DFB) LD at $1651 \mathrm{~nm}$. The seed was amplified by the optical parametric process in the crystal. Output lights were filtered out so that only the amplified $1651 \mathrm{~nm}$ was used for the methane measurement. Reflective tape is used as a target in the ground demonstration. A topographical ground is used for the airborne demonstration. The reflected photons were collected by a $20-\mathrm{cm}$ Cassegrain telescope and sent to the detector. We used a PIN detector and a photo-multiplier (PMT) for the ground and airborne measurements, respectively. Figure 2 (left) shows a photograph of the OPA system. All of the main optics were mounted on spring-free mounts, and further glued down to the baseplate for mechanical stability.

\subsection{OPA components}

The pump source is a passively Q-switched, Nd:YAG non-planar ring oscillator (NPRO) made by Innolight Inc. (Germany) that emits single-frequency, single longitudinal-mode output at $1064.5 \mathrm{~nm}$. It has $3.3-\mathrm{nsec}$ pulse width and $60-\mu \mathrm{J}$ energy at about $6-\mathrm{kHz}$ repetition rate. The optical linewidth of the NPRO is transform limited $(\sim 133 \mathrm{MHz})$. The seed source is a continuous-wave, polarization-maintaining fiber-coupled DFB LD. By tuning its injection current, the output signal can be tuned without a mode hop across the absorption peak of methane at $1651 \mathrm{~nm}$. The two laser beams are focused down to a crystal by a common lens. The beam radii inside the crystal are $\sim 100 \mu \mathrm{m}$ and $\sim 125 \mu \mathrm{m}$ for the pump and the seed, respectively. With this pump beam size and energy, the crystal is operated near the threshold of optical parametric generation. The nonlinear crystal is 50-mm long, $1-\mathrm{mm}$ thick MgO:PPLN. The end surfaces of the crystal were angled at $5^{\circ}$ and anti-reflective coated. We used $31.0-\mu \mathrm{m}$ grating at $128.4^{\circ} \mathrm{C}$.

\subsection{Characteristics of OPA output}

With a $60-\mu \mathrm{J}$ pump and a $15-\mathrm{mW}$ seed at $1651 \mathrm{~nm}$, we obtained $\sim 13-\mu \mathrm{J}$ signal output. The OPA output had $\sim 2$-nsec width and a distorted Gaussian pulse shape. The $\mathrm{M}^{2}$ of the OPA output signal beam was $\sim 1.3$. Using measurements with a gas cell, the OPA's output linewidth was estimated to be $\sim 500 \mathrm{MHz}$ when averaged over the beam. The effect of the broadened linewidth on the measured mixing ratio was small, since a typical full-width half maximum (FWHM) linewidth of our target line is $\sim 5 \mathrm{GHz}$ under Earth's atmospheric pressure. 


\section{GROUND DEMONSTRATION}

\subsection{Measurement arrangement}
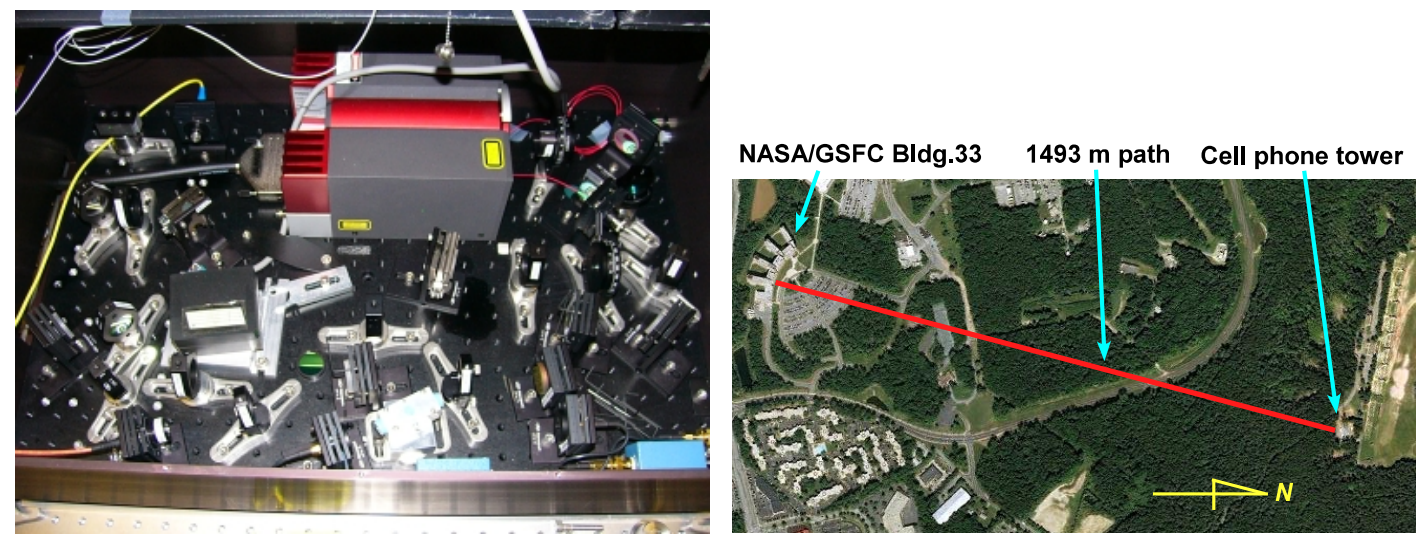

Figure 2. (Left) Photograph of the OPA breadboard. (Right) Aerial view of the open path.

For the ground demonstration, we set a cooperative hard target at $1493 \mathrm{~m}$ away, as shown in Figure 2 (right). The metal target was covered by automotive reflective tape and set on a cell phone tower. The reflected signal was detected by an InGaAs PIN detector. The pulsed signal from the detectors was averaged by boxcar averagers triggered by the residual pump pulse. In order to obtain temperature and atmospheric pressure measurements, which are needed to convert the measured absorption curve into mixing ratios, a weather station was set up outside. An in-situ cavity ring down spectrometer built by Picarro Inc. (USA) monitored the methane, carbon dioxide, and water mixing ratios of the outside air. The lidar system and the in-situ system were set up at Building 33 at GSFC, in Greenbelt, Maryland (USA).

The calculation of the mixing ratio was done by fitting the scan result with the theoretical Voigt profile. The DFB laser was continuously scanned by a ramp voltage at $2-\mathrm{Hz}$ rate. From the scan data, the baseline was determined by fitting to a polynomial function. The measured temperature, pressure, and round-trip path lengths were used to calculate the theoretical profile. Line parameters (center wavelength, line strength, etc.) were taken from the HITRAN 2008 database [8].

\subsection{Ground demonstration results}

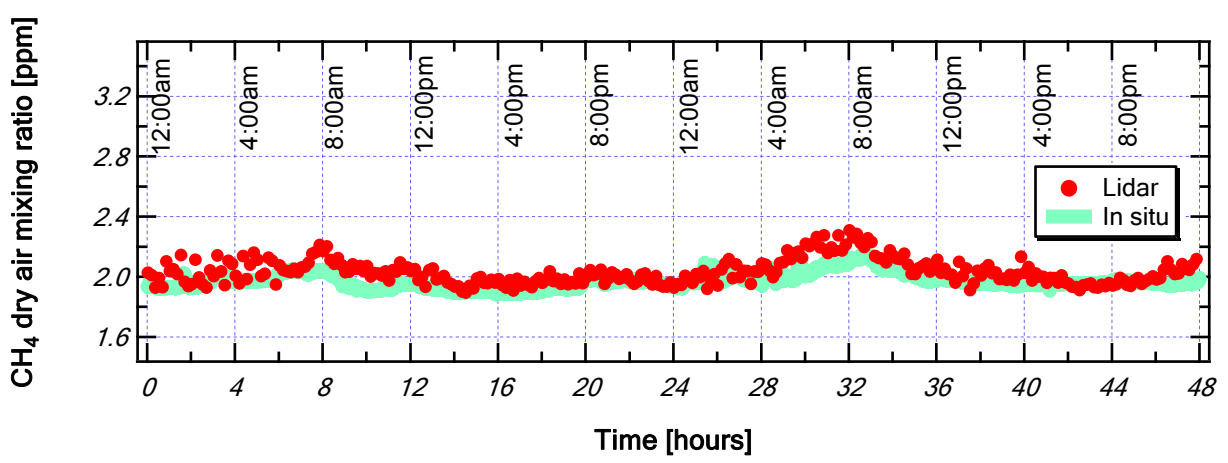

Figure 3. Measured diurnal variations of atmospheric $\mathrm{CH}_{4}$.

Figure 3 shows the results of the $\mathrm{CH}_{4}$ mixing ratio measured for 48 hours. The lidar and the in-situ sensor detected similar variations in the mixing ratios of the atmospheric methane. Their deviation can be explained by the difference between the lidar's column measurement and the in-situ device's point measurement. This result ensured that the OPA lidar system can monitor diurnal variations of methane on the ground. 


\section{AIRBORNE DEMONSTRATION}

\subsection{Measurement arrangement}
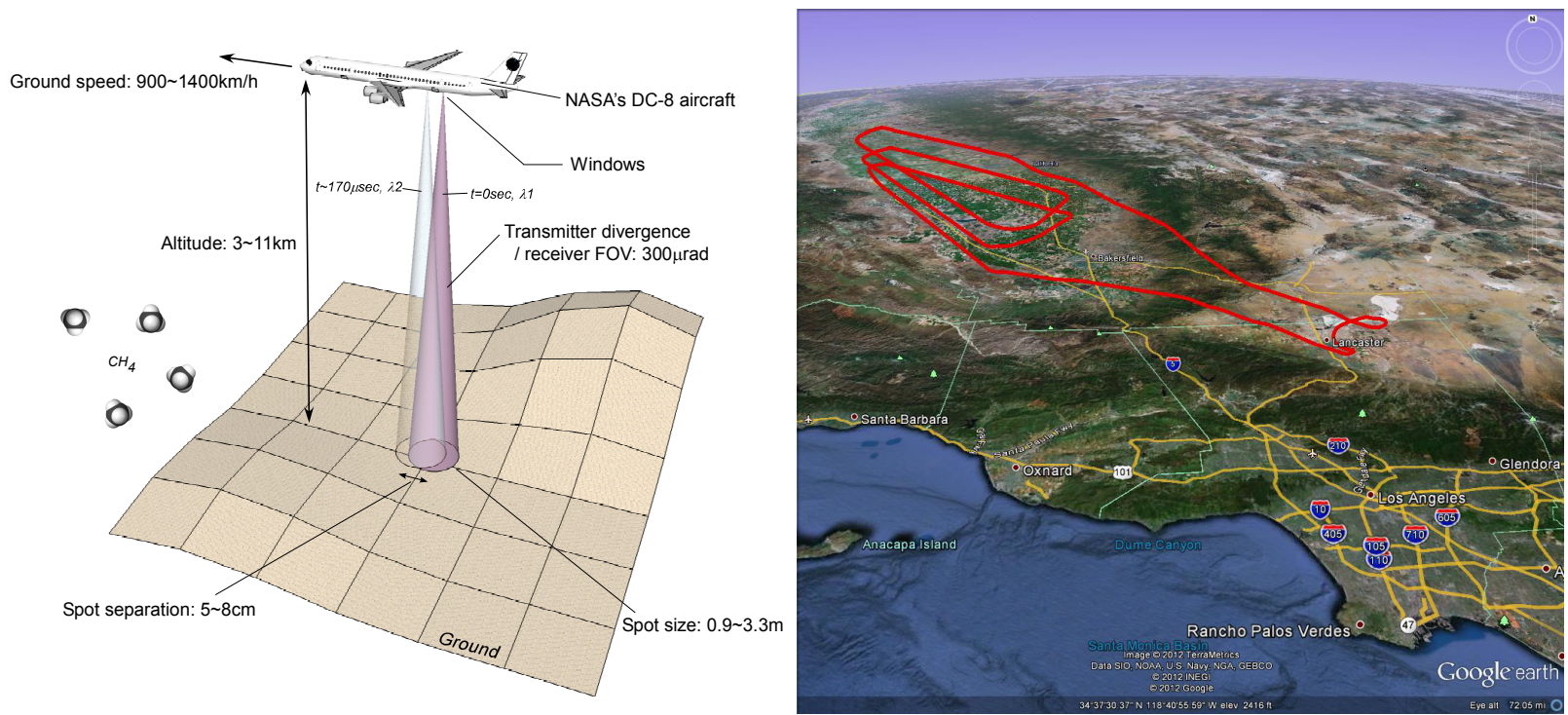

Figure 4. (Left) Schematic view of the airborne demonstration. FOV: field of view. (Right) Flight track of one of the test flights over the Central Valley, California (USA).

For the airborne demonstration, the InGaAs PIN detector was replaced by a near-infrared photo-multiplier (PMT), which detects a return signal from the earth's surface. The receiver was a commercial $20 \mathrm{~cm}$ diameter telescope whose output was coupled into a $600 \mu \mathrm{m}$ multi-mode fiber, filtered by a narrow-band optical filter, and then directed onto the PMT. The full divergence of the transmitter beam and the receiver telescope's field of view were adjusted to be $\sim 300 \mu \mathrm{rad}$. Two optical AR-coated windows were attached to the nadir port of the aircraft (DC-8 operated by NASA).

Since the pump laser (and hence the OPA signal output) was passively Q-switched, having short pulse width and high peak power, the PMT cannot be efficiently used in the digital (photon-counting) mode. There were multiple (1 10) return photons within the pulse width of $\sim 2 \mathrm{nsec}$, which was shorter than the pulse-pair resolution of the PMT. Therefore, we used the analog mode of PMT with relatively low high-voltage, and integrated the return pulse by the boxcar averager (as we did for the ground demonstration). To cope with the random timing of the outgoing pulse (due to the passive-Q nature) and the return pulse (due to the changing altitude), the timing for the integration and the wavelength scan were controlled by a field-programmable gate array (FPGA). Instead of the continuous seed wavelength scan, the seed (i.e., signal) wavelength was step-scanned by the FPGA, which was triggered by the (near random) pump pulse. We sampled 20 wavelength points across the $1650.9-\mathrm{nm} \mathrm{CH}_{4}$ line pulse by pulse.

As shown in Figure 4 (left), the altitude of the aircraft varied from $3 \mathrm{~km}$ to $11 \mathrm{~km}$. The resultant beam spot size was 0.9 $\mathrm{m} \sim 3.3 \mathrm{~m}$ on the ground. There were about $\sim 300$ scans in 1 second. The 1 -sec data was accumulated by the FPGA and saved in the computer, together with the round-trip length estimated from the pulse timing. The same in-situ instrument used for the ground demonstration was put on board to monitor gas concentrations around the aircraft. The airplane provided ancillary data such as GPS position, pressure, temperature, and humidity. The measurement campaign was performed over the Central Valley, California (USA) in August 2011. Figure 4 (right) shows the track of one of the flights. 


\subsection{Airborne demonstration results}
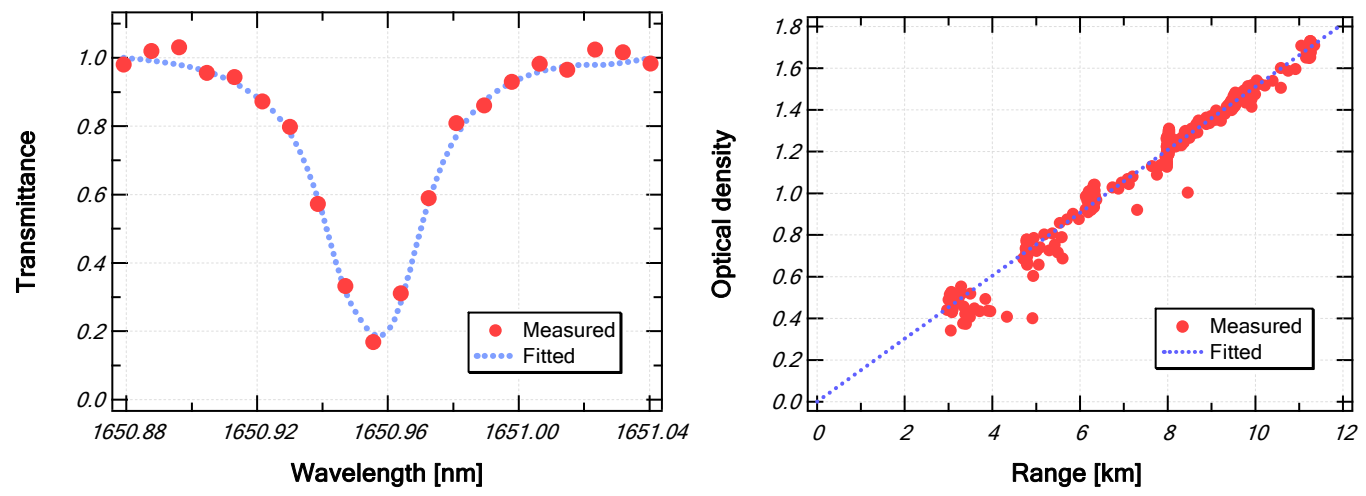

Figure 5. (Left) Example of measured scan data at 11.2-km range and fitted result. The transmittance was normalized at the edge wavelengths. (Right) The relationship between the measured optical density and the range.

The measured data was fitted to the theoretical profile, using the measured on-board data (temperature, pressure, $\mathrm{CO}_{2} / \mathrm{H}_{2} \mathrm{O}$ mixing ratio, wavelength), and modeled atmospheric data (surface temperature, pressure, and $\mathrm{H}_{2} \mathrm{O}$ mixing ratio). In the example shown in Figure 5 (left), the vertically uniform $\mathrm{CH}_{4}$ mixing ratio was used as a fitting parameter. To reduce statistical error, $10 \mathrm{sec}$ of the digitized data were averaged before the fitting. The $11.2-\mathrm{km}$ range was split in $200 \mathrm{~m}$ vertical layers in order to derive the theoretical fitting curve. Each layer was assumed to have the Voigt profile. As shown in Figure 5 (right), the measured optical density and the range were linearly correlated as expected. (The range is the distance between the aircraft and the ground as measured by the lidar.)
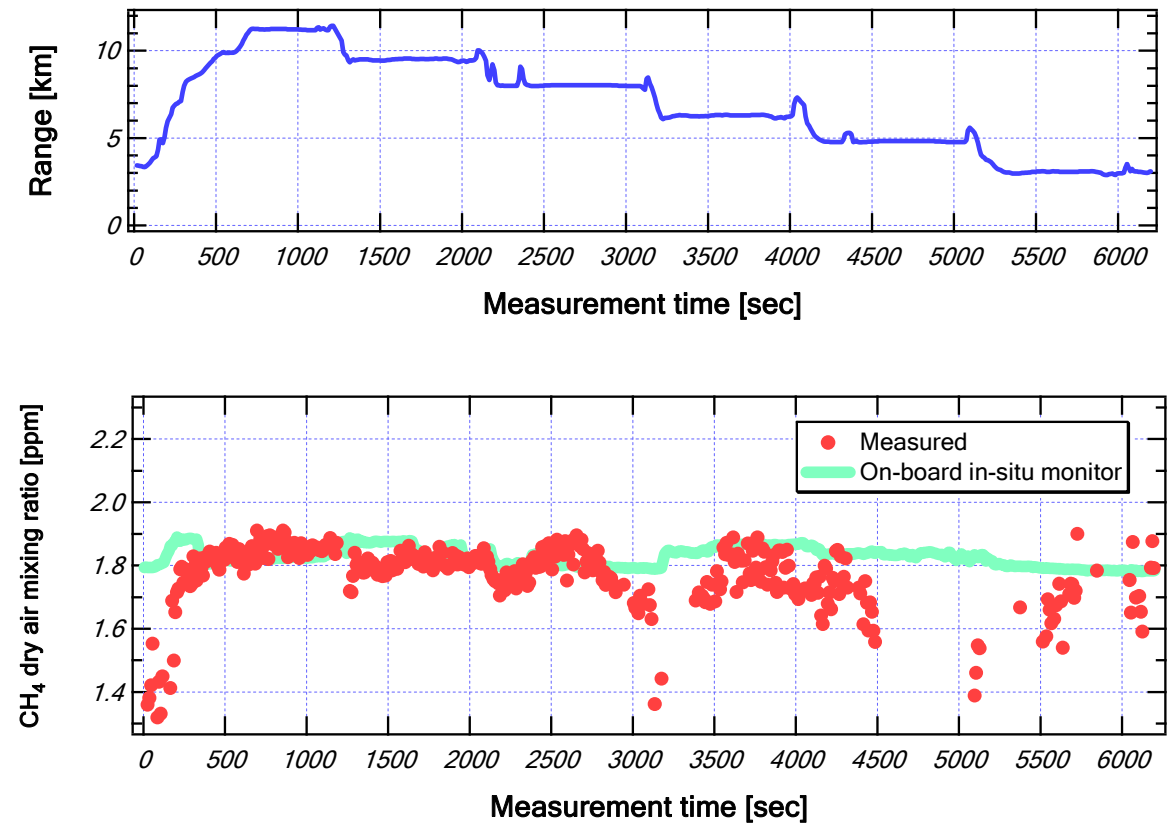

Figure 6. (Top) Range of the measurement. (Bottom) Derived $\mathrm{CH}_{4}$ dry air mixing ratio from the OPA lidar data and the measured result with the on-board in-situ monitor (ppmv).

Figure 6 shows the range (top) and the calculated $\mathrm{CH}_{4}$ dry air mixing ratio (bottom) as a function of time. For this flight, the retrieved $\mathrm{CH}_{4}$ favorably compared with the on-board in-situ monitor until time $\sim 3000 \mathrm{sec}$, between the 11 and $8 \mathrm{~km}$ range. After that, the cabin temperature variation caused misalignment between the transmitter and the receiver. It also caused uncorrectable instrument fringe variation. 


\section{CONCLUSIONS}

Global methane observations are highly desirable in order to understand climate change. We demonstrated the detection of methane on the ground and from an airplane using an OPA transmitter at $1.6 \mu \mathrm{m}$. More instrument system improvements are needed to enable stable data acquisition and true $\mathrm{CH}_{4}$ retrieval. However, the clean signal at $\sim 11-\mathrm{km}$ altitude shows that the OPA laser source and the measurement concept are very promising for spaceborne trace gas measurements. We will continue to improve the system, as well as to scale up the output power by $>10$ times, for spaceborne instruments.

This work is supported by the NASA Astrobiology Program's Astrobiology Science and Technology Instrument Development (ASTID) Program.

\section{REFERENCES}

[1] J. Lelieveld, P. J. Crutzen, and F. J. Dentener, "Changing concentration, lifetimes and climate forcing of atmospheric methane," Tellus B, 50, 128 (1998).

[2] Intergovernmental Panel on Climate Change Report (IPCC), 2007, Available at: http://www.ipcc.ch/index.htm.

[3] F. Keppler, J. T. G. Hamilton, M. Braß, T. Röeckmann, "Methane emissions from terrestrial plants under aerobic conditions," Nature, 439, 187 (2006).

[4] J. Houghton et al., Eds., "Climate Change 2001: The Scientific Basis," Cambridge Univ. Press, Cambridge, (2001), ISBN-10: 0521014956.

[5] Greenhouse gases Observing SATellite "IBUKI" (GOSAT), http://www.jaxa.jp/projects/sat/gosat/index e.html.

[6] C. Stephan, M. Alpers, B. Millet, G. Ehret, P. Flamant and C. Deniel, "MERLIN: a space-based methane monitor," Proc. SPIE 8159, 815908 (2011).

[7] A. Fix, G. Ehret, A. Hoffstädt, H. H. Klingenberg, C. Lemmerz, P. Mahnke, "Charm - a Helicopter-Borne LIDAR System for Pipeline Monitoring," 22nd Internation Laser Radar Conference (ILRC 2004), Proceedings of the Conference held 12-16 July, 2004 in Matera, Italy. Edited by Gelsomina Pappalardo and Aldo Amodeo. ESA SP-561. Paris: European Space Agency, 2004, p.45.

[8] L. S. Rothman, et. al., "The HITRAN 2008 molecular spectroscopic database," Journal of Quantitative Spectroscopy and Radiative Transfer, 110, 533 (2009). 